\title{
PD-L1 expression and the immune microenvironment in primary invasive lobular carcinomas of the breast
}

\author{
Elizabeth D Thompson ${ }^{1}$, Janis M Taube ${ }^{2}$, Rebecca J Asch-Kendrick ${ }^{1}$, Aleksandra Ogurtsova ${ }^{2}$, \\ Haiying $\mathrm{Xu}^{2}$, Rajni Sharma ${ }^{1}$, Alan Meeker ${ }^{1,3}$, Pedram Argani ${ }^{1,3}$, Leisha A Emens ${ }^{3}$ and \\ Ashley Cimino-Mathews ${ }^{1,3}$ \\ ${ }^{1}$ Department of Pathology, The Johns Hopkins Hospital, Johns Hopkins University School of Medicine, \\ Baltimore, MD, USA; ${ }^{2}$ Department of Pathology and Dermatology, The Johns Hopkins Hospital, Johns Hopkins \\ University School of Medicine, Baltimore, MD, USA and ${ }^{3}$ Department of Oncology, The Sidney Kimmel \\ Comprehensive Cancer Center, The Johns Hopkins Hospital, Johns Hopkins University School of Medicine, \\ Baltimore, MD, USA
}

\begin{abstract}
Tumor-infiltrating lymphocytes and immune checkpoint proteins such as PD-L1 are potential prognostic factors and therapeutic targets in breast cancer. Most studies characterizing the breast tumor immune microenvironment have focused on ductal carcinomas. Here we investigate the tumor microenvironment of primary invasive lobular carcinomas. Previously constructed tissue microarrays of 47 lobular carcinomas were labeled by immunohistochemistry for PD-L1, CD8, CD20, and FoxP3. The stromal immune infiltrate density was qualitatively scored as a percentage of tumor area: $1+(<5 \%)$; $2+(5-10 \%)$; $3+(10-15 \%)$; or $4+(>50 \%)$. The average immune cell subtype per high-power field was quantitatively scored. The percentage PD-L1 labeling on tumor-infiltrating lymphocytes was scored as none, focal $(<5 \%)$, moderate $(10-24 \%)$, or diffuse $(50-100 \%)$. The percentage of membranous carcinoma cell PD-L1 labeling was also recorded, with $<5 \%$ considered negative. All lobular carcinomas contained PD-L1+ tumor-infiltrating lymphocytes with the majority showing 1+ immune infiltrates with focal-moderate PD-L1 labeling. PD-L1 was expressed by tumor cells in $17 \%$ of lobular carcinomas. In contrast to ductal carcinomas, there was no correlation between the immune infiltrate density, the PD-L1 expression by lobular carcinoma cells, tumor grade, or the expression of estrogen receptor or human epidermal growth factor receptor-2. However, both the tumor-infiltrating lymphocyte density and the average CD8 ${ }^{+} \mathrm{T}^{-c e l l}$ counts correlated with immune cell PD-L1 status $(P=0.004$ and 0.03 , respectively). Similar to breast ductal carcinomas, PD-L1+ lobular breast carcinomas had higher numbers of PD-L1+ tumor-infiltrating lymphocytes $(63 \%)$ than PD-L1- lobular carcinomas $(23 \% ; P=0.04)$. These data show that a subset of primary breast lobular carcinomas both express PD-L1 on tumor cells and contain PD-L1 ${ }^{+}$tumor-infiltrating lymphocytes, suggesting the possibility of both constitutive and adaptive PD-L1 expression. Together, these results support immunotherapy as a potential treatment for a subset of patients with primary invasive lobular breast carcinomas. Modern Pathology (2017) 30, 1551-1560; doi:10.1038/modpathol.2017.79; published online 21 July 2017
\end{abstract}

Correspondence: Dr LA Emens, MD, PhD, Department of Oncology, The Sidney Kimmel Comprehensive Cancer Center at Johns Hopkins, The Johns Hopkins Hospital, Johns Hopkins University School of Medicine, Bunting-Blaustein Cancer Research Building, 1650 Orleans Street, Room 409, Baltimore, MD 21231-1000, USA. E-mail: emensle@jhmi.edu

Dr A Cimino-Mathews, MD, Departments of Pathology and Oncology, The Sidney Kimmel Comprehensive Cancer Center at Johns Hopkins, The Johns Hopkins Hospital, Johns Hopkins University School of Medicine, Weinberg Building 2242, 401N. Broadway Street, Baltimore, MD 21287, USA.

E-mail: acimino1@jhmi.edu

Received 20 January 2017; revised 24 May 2017; accepted 25 May 2017; published online 21 July 2017
The host immune response has an important role in breast cancer progression and response to therapy. Multiple studies in invasive ductal carcinomas have demonstrated improved survival and response to neoadjuvant therapy in patients with higher numbers of intratumoral cytotoxic T cells and B cells. ${ }^{1,2}$ Conversely, shorter progression-free and overall survival are associated with increased FoxP3 $^{+}$ regulatory $\mathrm{T}$ cells and a lower ratio of $\mathrm{CD}^{+} \mathrm{T}$ cells to FoxP3 ${ }^{+} \mathrm{T}$ cells. ${ }^{3}$ Triple-negative and human epidermal growth factor receptor-2-positive (HER-2 ${ }^{+}$) tumors show particularly strong correlations between tumor-infiltrating lymphocytes, survival, 
and response to therapy. Estrogen receptor-negative $\left(\mathrm{ER}^{-}\right)$and HER-2 ${ }^{+}$carcinomas harbor larger numbers of tumor-infiltrating lymphocytes than ER ${ }^{+}$tumors. ${ }^{4}$ Patients with triple-negative carcinomas and high tumor-infiltrating lymphocytes have longer survival, ${ }^{5,6}$ decreased distant recurrence, ${ }^{6,7}$ and increased time to relapse. ${ }^{8}$ Further, triple-negative carcinomas and $\mathrm{ER}^{-}$carcinomas with high tumorinfiltrating lymphocytes show improved responses to chemotherapy. 9,10

The association of a robust immune response in breast carcinomas with survival and treatment response suggests that enhancing the antitumor immune response may improve outcomes. Of particular interest is using immune checkpoint blockade, especially blockades targeting the pathway governed by programmed cell death protein-1 (PD-1) and its ligand programmed cell death ligand 1 (PD-L1). ${ }^{11,12}$ PD-1 (B71 ) is a member of the B7-CD28 family of co-stimulatory molecules and primarily binds to PD-L1 and PD-L2, which are inducible on the surface of tumor cells and tumor-infiltrating immune cells by interferon- $\gamma$ (IFN- $\gamma$ ) and other cytokines. ${ }^{13}$ In immune responses to infection, the PD-1/PD-L1 pathway serves as a feedback mechanism to prevent excessive T-cell activity and autoimmunity. However, in malignancy, PD-L1 upregulation in the tumor microenvironment counterregulates the antitumor immune response and can prevent effective antitumor immunity. PD-L1 is expressed on invasive breast carcinoma cells and their associated immune infiltrates. ${ }^{14-16}$ PD-L1 expression in breast carcinomas is associated with a higher number of tumor-infiltrating lymphocytes, ER negativity, and poor chemotherapy response. ${ }^{17}$ We previously reported PD-L1 labeling and patterns of tumor-infiltrating lymphocytes across the continuum from in situ, invasive, and metastatic ductal carcinomas. In both in situ and invasive ductal carcinomas, we observed more brisk lymphocytic infiltrates with more extensive PDL1 labeling in $\mathrm{ER}^{-}$tumors than in $\mathrm{ER}^{+}$tumors. ${ }^{12,18,19}$ PD-L1 labeling by invasive ductal carcinoma cells was also enhanced in $\mathrm{ER}^{-}$tumors. ${ }^{12,18}$

Previous studies have focused almost exclusively on invasive ductal carcinomas, with limited attention to the microenvironment of special subtypes of breast carcinoma. Recent molecular studies suggest a role for the immune microenvironment in some lobular carcinomas, which are the most common special histologic subtype of breast cancer. Two distinct molecular signatures of lobular carcinomas have been described: 'hormone related,' showing epithelial to mesenchymal transition, active ER and PR signaling, gains of chromosomes $1 q$ and $8 q$, and loss of 11q; and 'immune related,' showing upregulated expression of $P D-L 1, P D-1$, and $C T L A-4$ genes, high levels of expression of $C D 4$ and $C D 8$, cytokine and chemokine signaling pathways, and increased sensitivity to DNA-damaging agents. ${ }^{20}$ In addition, lobular carcinomas show increased B7H4 protein expression, ${ }^{21}$ increased PDL1 mRNA expression, ${ }^{22}$ and an association between B7H3 expression, IL-10 expression, and advanced stage. ${ }^{23}$ These findings suggest a potential role for immune suppression and immune resistance in a subset of lobular carcinomas.

Despite these findings, data characterizing the immune microenvironment of lobular carcinoma are limited. Here we profile the tumor-infiltrating lymphocyte composition and PD-L1 labeling patterns of 47 cases of primary invasive lobular carcinomas with defined ER, PR, and HER-2 status to explore whether a subset might display a similar inflamed immune microenvironment as their ductal counterparts.

\section{Materials and methods}

\section{Case Selection and Tissue Microarray Construction}

We constructed tissue microarrays $(1.4 \mathrm{~mm}$ cores, 5-10 cores per tumor that included a portion of adjacent normal tissue) from archived paraffin tissue blocks of 47 cases of primary infiltrating lobular carcinoma and associated lobular carcinoma in situ or atypical lobular hyperplasia as previously described. ${ }^{24,25}$ Briefly, all cases were incident lobular carcinomas surgically resected at our institution, and the morphologic impression of invasive lobular carcinoma was subsequently confirmed by loss of membranous E-cadherin labeling by immunohistochemistry at the time of diagnosis. The first 34 cases were identified as consecutive invasive lobular carcinomas, and $97 \%$ were $\mathrm{ER}^{+} / \mathrm{HER}-2^{-}$. The subsequent 12 cases were selected from the archives as consecutively identified triple-negative or HER-2 ${ }^{+}$ lobular carcinomas. If the primary invasive tumor was a localized mass, regions of both the periphery and center were included in the tissue microarray. Tissue microarrays were previously labeled for ER, PR, and HER-2 to subclassify cases into the following groups: $\mathrm{ER}^{+} / \mathrm{PR}^{+} / \mathrm{HER}-2^{-}$; triple-positive/luminal $\mathrm{B}$ $\left(\mathrm{ER}^{+} / \mathrm{PR}^{+} / \mathrm{HER}^{-}{ }^{+}\right)$; HER-2 ${ }^{+}\left(\mathrm{ER}^{-} / \mathrm{PR}^{-} / \mathrm{HER}^{-}{ }^{+}\right)$; and triple-negative $\left(\mathrm{ER}^{-} / \mathrm{PR}^{-} / \mathrm{HER}-2^{-}\right)$. ER and $\mathrm{PR}$ positivity was defined as $>1 \%$ nuclear labeling; HER-2 positivity was defined as $>10 \%$ complete strong membranous labeling or a fluorescence in situ hybridization ratio of $>2.0$, as per the 2012 ASCO/CAP guidelines. Clinicopathologic data, including gender, age, race, stage, progression, and survival are summarized in Table 1 . The median follow-up was 52 months (mean, 56 months; range, 0-167 months).

\section{Quantification of Tumor-infiltrating Lymphocytes}

We scored the density of stromal tumor-infiltrating lymphocytes on the hematoxylin-and-eosin-stained sections of the tissue microarrays in a qualitative manner as the percentage stromal area occupied by mononuclear lymphocytes per the recommended scoring guidelines of the International TILs Working Group. ${ }^{26,27}$ The density was scored as the average 
Table 1 Clinicopathologic characteristics of invasive lobular carcinoma cases

\begin{tabular}{|c|c|c|c|c|c|}
\hline & \multirow{2}{*}{ All cases } & \multicolumn{4}{|c|}{ Phenotype } \\
\hline & & $E R^{+} / H E R-2^{-}$ & $E R^{+} / H E R-2^{+}$ & $E R^{-} / H E R-2^{+}$ & $E R^{-} / H E R-2^{-}$ \\
\hline Total number $(n)$ & 47 & 33 & 8 & 1 & 5 \\
\hline \multicolumn{6}{|l|}{ Age (years) } \\
\hline Mean (range) & $59(34-87)$ & $58(34-79)$ & $62(41-78)$ & 87 & $62(43-84)$ \\
\hline \multicolumn{6}{|l|}{ Race } \\
\hline White & $37(79 \%)$ & $24(73 \%)$ & $8(100 \%)$ & $1(100 \%)$ & $4(80 \%)$ \\
\hline Black & $5(11 \%)$ & $5(15 \%)$ & $0(0 \%)$ & $0(0 \%)$ & $0(0 \%)$ \\
\hline Asian & $0(0 \%)$ & $0(0 \%)$ & $0(0 \%)$ & $0(0 \%)$ & $0(0 \%)$ \\
\hline Hispanic & $3(6 \%)$ & $2(6 \%)$ & $0(0 \%)$ & $0(0 \%)$ & $1(20 \%)$ \\
\hline Other & $2(4 \%)$ & $2(6 \%)$ & $0(0 \%)$ & $0(0 \%)$ & $0(0 \%)$ \\
\hline \multicolumn{6}{|l|}{ Grade } \\
\hline I & $5(11 \%)$ & $4(12 \%)$ & $1(13 \%)$ & $0(0 \%)$ & $0(0 \%)$ \\
\hline II & $34(72 \%)$ & $27(82 \%)$ & $6(74 \%)$ & $0(0 \%)$ & $1(20 \%)$ \\
\hline III & $8(17 \%)$ & $2(6 \%)$ & $1(13 \%)$ & $1(100 \%)$ & $4(80 \%)$ \\
\hline Mean tumor size $(\mathrm{cm})$ & 2.8 & 2.7 & 2.7 & 7.1 & 3.2 \\
\hline \multicolumn{6}{|l|}{ Stage } \\
\hline I & $16(34 \%)$ & $16(49 \%)$ & $0(0 \%)$ & $0(0 \%)$ & $0(0 \%)$ \\
\hline II & $20(43 \%)$ & $10(30 \%)$ & $7(87 \%)$ & $0(0 \%)$ & $3(60 \%)$ \\
\hline III & $11(23 \%)$ & $7(21 \%)$ & $1(13 \%)$ & $1(100 \%)$ & $2(40 \%)$ \\
\hline IV & $0(0 \%)$ & $0(0 \%)$ & $0(0 \%)$ & $0(0 \%)$ & $0(0 \%)$ \\
\hline \multicolumn{6}{|c|}{ Subsequent metastatic disease } \\
\hline Yes & $5(11 \%)$ & $1(3 \%)$ & $1(13 \%)$ & $1(100 \%)$ & $2(40 \%)$ \\
\hline No & $42(89 \%)$ & $32(97 \%)$ & $7(87 \%)$ & $0(0 \%)$ & $3(60 \%)$ \\
\hline \multicolumn{6}{|l|}{ Death due to disease } \\
\hline Yes & $4(9 \%)$ & $1(3 \%)$ & $1(13 \%)$ & $0(0 \%)$ & $2(40 \%)$ \\
\hline No & $43(91 \%)$ & $32(97 \%)$ & $7(87 \%)$ & $1(100 \%)$ & $3(60 \%)$ \\
\hline
\end{tabular}

Abbreviations: HER-2, human epidermal growth factor 2; LCIS, lobular carcinoma in situ.

stromal tumor-infiltrating lymphocytes. The scores were subdivided into the following categories: none (0); rare (1+; tumor-infiltrating lymphocytes comprising $<5 \%$ of tumor stromal area); mild $(2+$; tumor-infiltrating lymphocytes comprising $5-10 \%$ of tumor stromal area); moderate (3+; tumor-infiltrating lymphocytes comprising $11-49 \%$ of tumor stromal area); or brisk (4+; tumor-infiltrating lymphocytes comprising $>50 \%$ of tumor stromal area).

\section{Immunohistochemistry for Lymphocyte Subsets and PD-L1}

Using immunohistochemistry, we labeled tissue microarrays for cytokeratin AE1/AE3 (catalog no. mab3412, dilution 1:4000, Chemicon, Temecula, CA, USA) and CD45 (clone PD7/26+2b1, catalog no. m0701, Dako, Carpintera, CA, USA) to highlight the carcinoma cell and lymphocyte populations, respectively. Tumor-infiltrating lymphocyte subsets were identified by immunohistochemical labeling for CD8 (mouse monoclonal, clone C8/C8144B, catalog no. 760-4250, Cell Marque, Rockin, CA, USA), CD20 (monoclonal, clone MS/L26, catalog no. 760-2531, Ventana Medical Systems, Tucson, AZ, USA), and FoxP3 (mouse monoclonal, clone 236A/E7, catalog no. 14-4777-80, dilution 1:50, eBioscience, San
Diego, CA, USA) as previously described. ${ }^{12,19}$ We defined CD8 and CD20 expression as membranous lymphocyte cell labeling, and FoxP3 expression as nuclear lymphocyte cell labeling. We manually counted the number of CD8, CD20, and FoxP3 cells per one high-power field for each core and averaged each across cores from the same case of invasive lobular carcinoma. Each high-power field was chosen as representative of the overall tumor lymphocytic infiltration in the core.

We labeled tissue microarrays for PD-L1 (B7-H1) using the rabbit monoclonal anti-human PD-L1 antibody, SP142 (Spring Bioscience, Pleasanton, CA, USA). ${ }^{28}$ PD-L1 labeling was scored by two pathologists (EDT and ACM) blinded to patient clinicopathologic characteristics, and discrepancies were adjudicated by a third pathologist (JMT). We scored the percentage invasive lobular carcinoma cells with clear membranous PD-L1 labeling in 5\% increments, with any intensity labeling $>5 \%$ considered positive. We scored the percentage PD-L1 labeling by tumor-infiltrating lymphocytes as none (0), focal $(1 ;<5 \%)$, moderate $(2 ; 6-49 \%)$, or diffuse (3; 50-100\%). The PD-L1 labeling by carcinoma cells and TIL was scored as an average across all tissue microarray spots. 

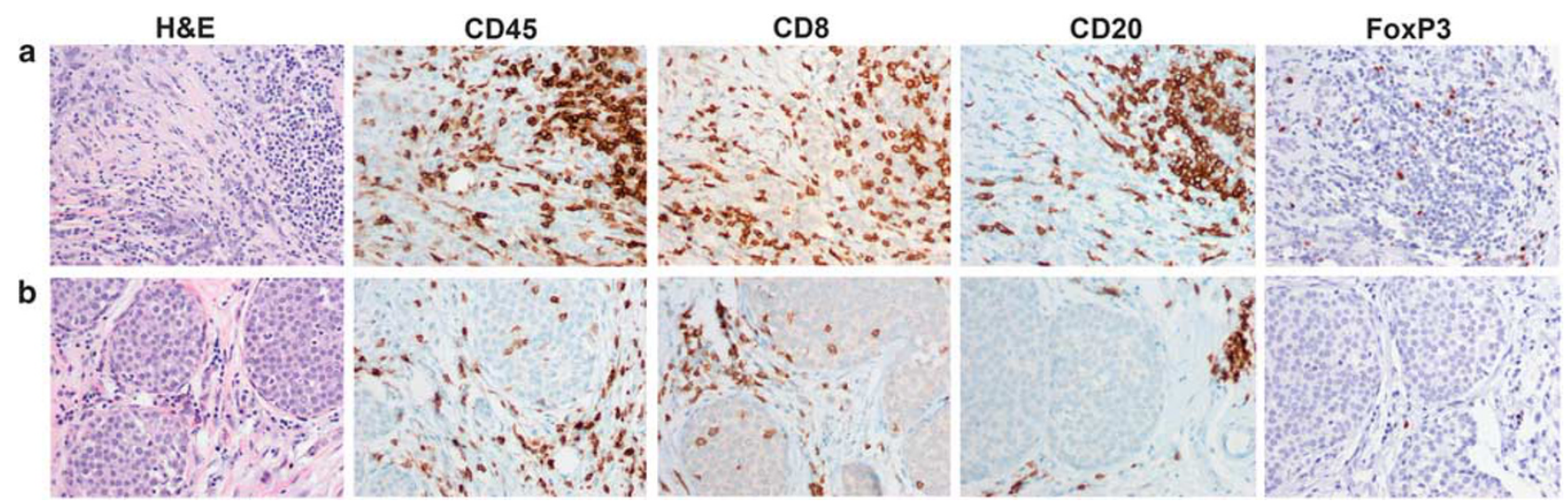

Figure 1 Immunologic features of the tumor microenvironment of invasive lobular carcinoma. (a) An active immune response highlighted by $\mathrm{CD}_{4} 5^{+}$lymphocytes is associated with a subset of invasive lobular carcinomas and includes $\mathrm{CD}^{+} \mathrm{T}$ cells, CD20 ${ }^{+} \mathrm{B}$ cells, and FoxP3 ${ }^{+}$ regulatory T cells. (b) Lobular carcinoma in situ (LCIS) associated with invasive lobular carcinomas also contains tumor-infiltrating lymphocytes, which are found both within the LCIS and around the periphery of the in situ nests.

\section{Statistical Analysis}

We performed statistical analysis using paired, two-tailed Student's $t$-test and the Fisher's exact test.

\section{Results}

Tumor-Infiltrating Lymphocytes Correlate with Patient Age and Race in Invasive Lobular Carcinoma

We first evaluated the density and composition of immune infiltrates associated with invasive lobular carcinomas. All tumors in our series contained tumorinfiltrating lymphocytes (Figure 1a). The majority $(53 \%)$ showed $1+$ infiltrates, and $32 \%$ and $15 \%$ contained $2+$ and $3+$ infiltrates, respectively. No invasive lobular carcinoma had 4+ brisk lymphocytic infiltration (defined as $>50 \%$ tumor stroma area). The average CD8 count per high-power field increased correspondingly across tumors with 1+, 2 + , and $3+$ qualitative tumor-infiltrating lymphocyte scores (from 23 to 40 to $88 \mathrm{CD}^{+}$cells per high-power field, respectively; $P<0.0002$ ). Associated regions of atypical lobular hyperplasia or lobular carcinoma in situ also showed tumor-infiltrating lymphocytes, both within the in situ lesions and at the interface between in situ lesion and normal breast and/or adjacent invasive lobular carcinoma (Figure 1b). Patients with 3+ tumor-infiltrating lymphocytes were younger (mean age, 46 years) than patients with $1+/ 2+$ tumor-infiltrating lymphocytes (mean age, 62 years; $P=0.001$; Table 2). By qualitative assessment on hematoxylin and eosin sections, invasive lobular carcinomas in Black patients had fewer tumorinfiltrating lymphocytes (100\% with 1+ lymphocytes) than invasive lobular carcinomas in White patients (46\% with $1+$ lymphocytes; $P=0.049$ ); there was a trend toward higher individual CD8 and CD20 cell counts and higher CD8/FoxP3 ratios in tumors from Black patients compared to White patients $(P=0.2-$ 0.3). There was no correlation between tumor- infiltrating lymphocyte density or individual immune cell counts by CD8, CD20, or FoxP3 and the ER status, HER-2 status, grade, size, or stage of the invasive lobular carcinomas.

\section{Tumor Cell PD-L1 Expression Correlates with Younger Patient Age but not Carcinoma Phenotype, Grade, or Outcome}

We next assessed the prevalence of PD-L1 labeling in lobular carcinoma cells. We found that $17 \%(8 / 47)$ of invasive lobular carcinomas (Figure 2a and b) and $14 \%(3 / 22)$ of associated atypical lobular hyperplasia/lobular carcinoma in situ showed strong membranous carcinoma cell PD-L1 labeling. Of all cases of atypical lobular hyperplasia/lobular carcinoma in situ, $77 \%$ displayed concordant PD-L1 labeling with the associated invasive component; however, there was one case in which the lobular carcinoma in situ cells were PD-L1 ${ }^{+}$but the associated invasive carcinoma cells were PD-L1 ${ }^{-}$. Patients with PD-L1 ${ }^{+}$ invasive lobular carcinomas were younger (mean age, 51 years) than patients with PD-L1 ${ }^{-}$invasive lobular carcinoma (mean age, 62 years) $(P=0.02)$. There was no correlation between the PD-L1 labeling of the invasive lobular carcinoma and the carcinoma phenotype (ie, hormone receptor or HER-2 status), grade, size, stage, patient race, or death due to disease.

\section{PD-L1 Expression by Tumor-infiltrating Lymphocytes Correlates with the Overall Degree of Lymphocyte Infiltration}

We next assessed PD-L1 expression on stromal tumor-infiltrating lymphocytes. All invasive lobular carcinomas contained PD-L1 ${ }^{+}$tumor-infiltrating lymphocytes, with $71 \%$ displaying focal or moderate labeling, and 29\% displaying diffuse labeling (ie, $>50 \%$ tumor-infiltrating lymphocytes labeling for PD-L1; Table 2). Tumors with greater numbers of 


\begin{tabular}{|c|c|c|c|c|c|c|c|c|c|c|c|c|c|}
\hline \multirow{2}{*}{ Immune parameters } & \multicolumn{2}{|c|}{ Age } & \multicolumn{2}{|c|}{ Race } & \multicolumn{4}{|c|}{ Phenotype } & \multicolumn{2}{|c|}{ Tumor PD-L1 } & \multicolumn{3}{|c|}{$\begin{array}{l}\text { Tumor-infiltrating lymphocyte } \\
\text { PD-L1 }\end{array}$} \\
\hline & All cases & Average & White & Black & $E R^{+} / H E R-2^{-}$ & $E R^{+} / H E R-2^{+}$ & $E R^{-} / H E R-2^{+}$ & $E R^{-} / H E R-2^{-}$ & Positive & Negative & Focal (1) & Mod. (2) & Diffuse (3) \\
\hline Total n (\%) & 47 & 59 & $37(79 \%)$ & $5(11 \%)$ & $33(70 \%)$ & $8(17 \%)$ & $1(2 \%)$ & $5(11 \%)$ & $8(17 \%)$ & $39(83 \%)$ & $5(11 \%)$ & $28(60 \%)$ & $14(30 \%)$ \\
\hline \multicolumn{14}{|c|}{ Tumor-infiltrating lymphocyte density } \\
\hline 0 (none) & 0 & NA & 0 & 0 & 0 & 0 & 0 & 0 & 0 & 0 & 0 & 0 & 0 \\
\hline $1+($ rare $)$ & $25(53 \%)$ & $64^{*}$ & $17(45 \%)^{*}$ & $5(100 \%)^{*}$ & $17(52 \%)$ & $5(63 \%)$ & $1(100 \%)$ & $2(40 \%)$ & $3(38 \%)$ & $22(36 \%)$ & $4(80 \%)^{*}$ & $20(71 \%)^{*}$ & $1(7 \%)^{*}$ \\
\hline $2+($ mild $)$ & $15(32 \%)$ & $58^{*}$ & 14 & 0 & $10(30 \%)$ & $2(25 \%)$ & 0 & $3(60 \%)$ & $3(38 \%)$ & $12(31 \%)$ & $1(20 \%)$ & $5(18 \%)$ & $9(64 \%)$ \\
\hline $3+$ (moderate) & $7(15 \%)$ & $46^{*}$ & 6 & 0 & $6(18 \%)$ & $1(12 \%)$ & 0 & 0 & $2(25 \%)$ & $5(13 \%)$ & $0^{*}$ & $3(11 \%)^{*}$ & $4(29 \%)^{*}$ \\
\hline $4+$ (brisk) & 0 & NA & 0 & 0 & 0 & 0 & 0 & 0 & 0 & 0 & 0 & 0 & 0 \\
\hline CD8/hpf & 37.9 & & 41.7 & 23.6 & 40.4 & 29.5 & 37 & 34.7 & 46.1 & 36.2 & $19.8^{*}$ & $30.2^{*}$ & $59.7^{*}$ \\
\hline CD20/hpf & 17.7 & & 20.9 & 6.67 & 17.6 & 19.7 & 13 & 15.8 & 13.8 & 18.5 & 3.3 & 19.4 & 19.4 \\
\hline FoxP3/hpf & 7.7 & & 8.8 & 7 & 8.5 & 6.5 & 10 & 4 & $13.3^{*}$ & $6.5^{*}$ & $2.2^{*}$ & $6.1^{*}$ & $12.8^{*}$ \\
\hline Mean CD8/FoxP3 & 10.4 & & 9.32 & 9.32 & 9.6 & 8.7 & 3.7 & 20 & 5.9 & 11.4 & 11.2 & 10.6 & 9.8 \\
\hline \multicolumn{14}{|l|}{ Tumor cell PD-L1 } \\
\hline Positive & $8(17 \%)$ & $51^{*}$ & $6(16 \%)$ & $1(20 \%)$ & $6(18 \%)$ & $1(13 \%)$ & 0 & $1(20 \%)$ & & $1(20 \%)$ & $2(7 \%)$ & $5(36 \%)$ & \\
\hline Negative & $39(83 \%)$ & $62^{*}$ & $31(84 \%)$ & $4(80 \%)$ & $27(82 \%)$ & $7(87 \%)$ & $1(100 \%)$ & $4(80 \%)$ & & $4(80 \%)$ & $26(93 \%)$ & $9(64 \%)$ & \\
\hline \multicolumn{14}{|c|}{ Tumor-infiltrating lymphocyte PD-L1 } \\
\hline Focal (1) & $5(11 \%)$ & 66 & 0 & $4(11 \%)$ & $5(15 \%)$ & 0 & 0 & 0 & $1(12 \%)$ & $4(10 \%)$ & & & \\
\hline Moderate (2) & $28(60 \%)$ & 59 & $5(100 \%)$ & $20(54 \%)$ & $18(55 \%)$ & $7(88 \%)$ & $1(100 \%)$ & $2(40 \%)$ & $2(25 \%)$ & $26(67 \%)$ & & & \\
\hline Diffuse (3) & $14(29 \%)$ & 57 & 0 & $13(35 \%)$ & $10(30 \%)$ & $1(12 \%)$ & 0 & $3(60 \%)$ & $5(63 \%)^{*}$ & $9(23 \%)^{*}$ & & & \\
\hline
\end{tabular}

Abbreviations: ER, estrogen receptor; HER-2, human epidermal growth factor receptor-2; mod., moderate; PD-L1, programmed death ligand 1.

Statistical analysis performed using Fisher's exact test or two-tailed Student's $t$-test.

${ }^{*} P$-value $<0.05$. 

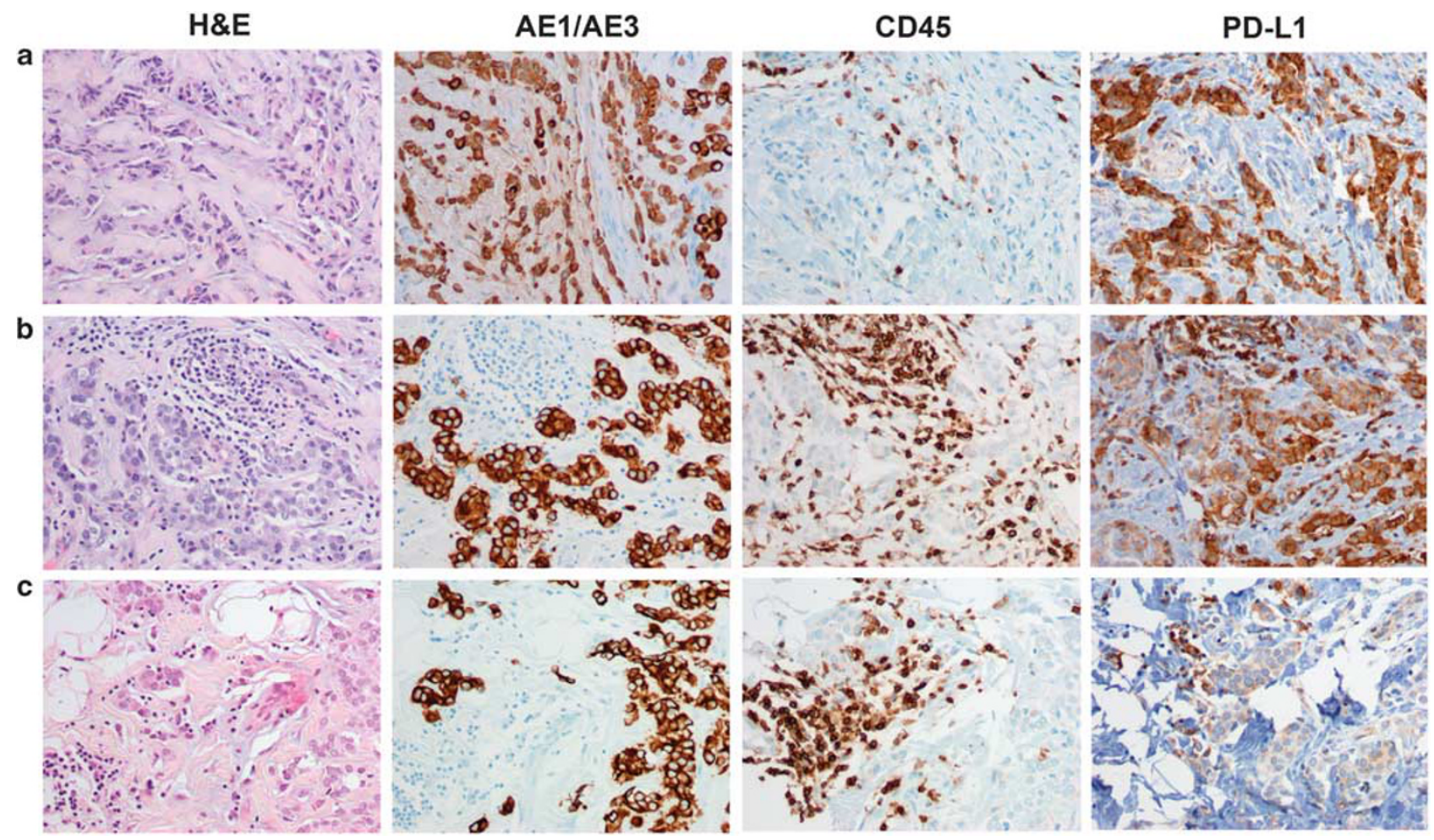

Figure 2 A subset of primary invasive lobular carcinomas express PD-L1 with patterns suggesting both adaptive and constitutive mechanisms of expression. Tumor cell PD-L1 expression does not correlate with hormonal status or density of tumor-infiltrating lymphocytes. Tumor cells are highlighted by cytokeratin AE1/AE3 immunostain, and lymphocytes are highlighted by CD45 immunostain. (a) An $\mathrm{ER}^{+}$lobular carcinoma with diffuse PD-L1 labeling despite the rare presence of tumor-infiltrating lymphocytes, suggesting a constitutive mechanism of tumor cell expression of PD-L1. (b) An ER ${ }^{+}$lobular carcinoma with PD-L1 labeling on both tumor and lymphocytes, in the presence of a moderate degree of lymphocyte infiltration suggests both adaptive and constitutive mechanisms of PD-L1 expression. (c) An ER- lobular carcinoma that lacks PD-L1 labeling in the presence of mild tumor-infiltrating lymphocytes and focal PD-L1 expression on immune cells only.

tumor-infiltrating lymphocytes had a greater proportion of PD-L1 ${ }^{+}$lymphocytes, in that $37 \%$ tumors with $3+$ tumor-infiltrating lymphocytes displayed diffuse PD-L1 positivity compared to $4 \%$ of tumors with $1+$ tumor-infiltrating lymphocytes $(P=0.004)$. Further, infiltrating lobular carcinomas with a high degree of PD-L1 ${ }^{+}$infiltrates had more $\mathrm{CD}^{+}$and $\mathrm{FoxP}^{+}{ }^{+} \mathrm{T}$ cells than tumors with focal to moderate PD-L1 ${ }^{+}$infiltrates $(P<0.03)$, but equivalent $\mathrm{CD} 8 / \mathrm{FoxP} 3$ ratios. This association of PD-L1 expression by the tumorinfiltrating lymphocytes with the overall degree of lymphocyte infiltrate and with higher average CD8 T-cell counts suggests a degree of IFN- $\gamma$-driven adaptive expression of PD-L1 within the lobular carcinoma microenvironment. Finally, we evaluated the association of PD-L1 expression on tumorinfiltrating lymphocytes with tumor hormone receptor status. ER ${ }^{-}$invasive lobular carcinomas had a higher proportion of PD-L1 ${ }^{+}$tumor-infiltrating lymphocytes $\left(50 \%\right.$ with diffuse ${\mathrm{PD}-\mathrm{L} 1^{+}}^{+}$infiltrates) compared to $\mathrm{ER}^{+}$invasive lobular carcinoma $(27 \%$ with diffuse PD-L1 ${ }^{+}$infiltrates), but this was not statistically significant $(P=0.3)$. All cases with focal $\mathrm{PD}-\mathrm{L}^{+}$tumor-infiltrating lymphocytes were in $\mathrm{ER}^{+}$ invasive lobular carcinomas.
Tumor Cell PD-L1 Expression Correlates with the Proportion of PD-L1+ Tumor-Infiltrating

Lymphocytes, but not with the Overall Degree of Lymphocyte Infiltration or the Tumor Cell ER/HER-2 Status

We next assessed the relationship between tumor cell PD-L1 expression and the degree of lymphocyte infiltration and lymphocyte PD-L1 expression. In contrast to the observed associations in ductal breast carcinomas, there was no correlation between PD-L1 labeling on the lobular carcinoma tumor cells and the density of the overall lymphocyte infiltrate, or with either the lobular carcinoma grade or ER/HER-2 status (Figure 2). However, there was a correlation between tumor cell PD-L1 labeling and the proportion of $\mathrm{PD}-\mathrm{L}^{+}$lymphocytes in the tumor microenvironment, such that $63 \%$ of lobular carcinomas with $\mathrm{PD}-\mathrm{L}^{+}{ }^{+}$tumor cells contained a high proportion of PD-L1 $1^{+}$lymphocytes compared to $23 \%$ of lobular carcinomas with PD-L1 ${ }^{-}$tumor cells $(P=0.04)$. Taken together, these data support both constitutive and adaptive PD-L1 expression within the lobular breast carcinoma immune microenvironment. 


\section{Discussion}

Our data demonstrate several important characteristics of the immune milieu in invasive lobular breast carcinoma. First, all lobular carcinomas in this cohort contained tumor-infiltrating lymphocytes, with a subset showing a high proportion of PD-L1 ${ }^{+}$ lymphocytes. Second, a subset of invasive lobular carcinomas $(17 \%)$ shows expression of PD-L1 by lobular carcinoma cells. Similar to ductal carcinoma in situ, lobular carcinoma in situ/atypical lobular hyperplasia, when present, also harbors an associated immune infiltrate that tends to reflect the PDL1 labeling pattern of the associated invasive lobular carcinoma. In contrast to our previous studies of invasive ductal carcinomas, we found no correlation between carcinoma cell PD-L1 labeling and the overall density of the lymphocyte infiltration, ER status, HER-2 status, or tumor grade of the invasive lobular carcinomas. However, we did find that PD-L1 labeling on invasive lobular carcinomas correlates with a higher proportion of PD-L1 ${ }^{+}$tumor-infiltrating lymphocytes, and that the presence of higher numbers of PD-L1 ${ }^{+}$tumor-infiltrating lymphocytes correlates both with the overall degree of lymphocytic infiltration and with higher $\mathrm{CD}^{+} \mathrm{T}$-cell counts.

Our study highlights distinct differences between the immune microenvironment of invasive ductal carcinomas and invasive lobular carcinomas of the breast. We and others have shown a correlation between increasing density of tumor-infiltrating lymphocytes and ER negativity in both invasive ${ }^{4,17}$ and in situ ductal carcinoma. ${ }^{19}$ PD-1/PD-L1 expression on both tumor-infiltrating lymphocytes and tumor cells is also higher in $\mathrm{ER}^{-}$ductal carcinomas than in $\mathrm{ER}^{+}$ductal carcinomas. ${ }^{15,17,29}$ In contrast, in our cohort of invasive lobular carcinomas, there was no association between tumor-infiltrating lymphocyte density or hormone receptor status and the PDL1 labeling in carcinoma cells. In one prior study, $\mathrm{CD}^{+}$, $\mathrm{FoxP}^{+}$, and $\mathrm{IL}_{-1}-7^{+}$tumor-infiltrating lymphocytes in invasive breast carcinomas were increased in ductal carcinomas as compared to lobular carcinomas, and were associated with ER negativity in ductal but not in lobular tumors. ${ }^{30}$ This report is consistent with our observation that the lymphocyte density and tumor cell PD-L1 labeling in lobular carcinomas do not track together. These findings suggest that different pathways may dominate the regulation of PD-L1 expression in ductal and lobular breast carcinomas.

PD-L1 upregulation in the tumor microenvironment can occur via adaptive or constitutive pathways. In the former, expression is driven by IFN- $\gamma$ and toll-like receptor signaling generated by activated immune cells, ${ }^{31-34}$ whereas constitutive expression is due to oncogenic signaling. The PDL1/PD-1 pathway serves as one of the multiple mechanisms of immune regulation to restrain immune responses and regulate their propagation. The induction of PD-L1 expression within tumors has been termed adaptive immune resistance, reflecting the ability of tumors to escape the active antitumor immune response through upregulation of the PD-1 immune checkpoint pathway in response to inflammatory stimuli. ${ }^{35}$ Adaptive immune resistance has been described in an array of tumors, including melanoma, Merkel cell carcinoma, non-small-cell lung cancers, and breast carcinoma. ${ }^{4,35-37}$

Most prior studies in ductal carcinomas of the breast support a dominant role for adaptive pathways of PD-L1 expression, in which highly inflamed tumors, enriched for those lacking ER expression, demonstrate the highest rates of PD-L1 expression on both tumor cells and tumor-infiltrating lymphocytes. In contrast, in our study of primary lobular carcinomas, tumor-infiltrating lymphocytes of varying densities were distributed amongst $\mathrm{ER}^{-}$and $\mathrm{ER}^{+}$tumors. Diffuse PD-L1 expression by tumor-infiltrating lymphocytes was seen even in non-inflamed $\mathrm{ER}^{+}$tumors, suggesting that adaptive expression may not be the only driver of tumor cell PD-L1 upregulation in the lobular carcinoma microenvironment.

In addition to the adaptive pathway of PD-L1 upregulation, PD-L1 can also be upregulated in the tumor microenvironment via a constitutive pathway of expression related to intrinsic tumor cell mutations. The lack of correlation between PD-L1 labeling in invasive lobular carcinoma cells and the density of lymphocytic infiltration in these tumors suggests that other factors, such as oncogenic signaling via mutations intrinsic to carcinoma cells, may influence PD-L1 expression independent of cytokine modulation via tumor-infiltrating lymphocytes. Constitutive expression of PD-L1 has been reported in multiple tumor types associated with a variety of signaling pathways. ${ }^{38}$ For example, loss of PTEN function can lead to PD-L1 upregulation in glioblastoma ${ }^{39}$ and colorectal carcinomas $^{40}$ as a result of oncogenic mutation or microRNA inhibition, respectively. Chromosomal translocations and amplifications involving 9p24.1 lead to constitutive expression of PD-L1 in some lymphomas, ${ }^{41,42}$ and 9p24.1 amplification has been described in triplenegative breast carcinomas. ${ }^{43}$ In addition, PD-L1 expression in certain T-cell lymphomas can result from signaling through the tyrosine kinase NPMALK via STAT3 signaling. ${ }^{44}$ Anaplastic large-cell lymphomas and Hodgkin lymphomas can both express PD-L1 as a result of ERK activation. ${ }^{45}$ In non-small-cell lung carcinomas, inhibition of epidermal growth factor receptor (EGFR) by erlotinib decreased PD-L1 expression in EGFR-mutated tumor cells, suggesting a possible link between EGFRmediated carcinogenesis and PD-L1 expression. ${ }^{46}$ Links between the epithelial-mesenchymal transition and PD-L1 expression have been suggested in mouse models through alterations in E-cadherin. ${ }^{46}$

In addition to constitutive expression, there was also evidence of adaptive PD-L1 expression. Specifically, while there was no association between overall tumor-infiltrating lymphocyte density and 
PD-L1 expression on tumor cells in lobular carcinoma, we did see an association between overall infiltrating immune cell density and average $\mathrm{CD}^{+}$ cell counts and PD-L1 expression on tumorinfiltrating lymphocytes. In addition, PD-L1 ${ }^{+}$lobular carcinomas were more likely to contain $\mathrm{PD}-\mathrm{L}^{+}$ tumor-infiltrating lymphocytes than PD-L1- ${ }^{-}$carcinomas. Our data support at least three patterns of PD-L1 expression in the lobular breast tumor microenvironment: adaptive; constitutive; and a combination, with both adaptive immune resistance and constitutive PD-L1 expression at play within individual tumors. This combination of regulatory mechanisms governing PD-L1 expression in lobular carcinoma suggests the possibility of combinatorial therapies targeting both immune-related and intrinsic oncogenic signaling pathways of upregulation.

This study is limited by the relatively small sample size and the low number of hormone receptor-negative cases of lobular breast carcinomas, although it should be noted that the majority of invasive lobular carcinomas are hormone receptorpositive. The tissue microarray platform is further limited by tumor sampling that may not reflect potential heterogeneity within an individual primary tumor. To address these concerns, we sampled five large cores per individual tumor, roughly estimating the amount of tumor material present on a small core needle biopsy. A recent study has shown that core needle biopsies are adequate in assessing the overall tumor-infiltrating lymphocyte density in tumors. ${ }^{47}$ In addition, the tissue microarray cores were sampled from both the periphery and center of the tumor to address any potential spatial heterogeneity. Notably, concerns about spatial heterogeneity are mitigated as most invasive lobular carcinomas infiltrate through the benign breast parenchyma in a permeative manner, such that there is rarely a distinct 'leading edge' as seen in invasive ductal carcinoma.

In conclusion, our data demonstrate an active tumor immune microenvironment in invasive lobular breast carcinomas with data supporting both adaptive and constitutive expression of PD-L1 in a pattern distinct from that seen in ductal breast carcinomas. Together with our previously published data, this study supports further exploration of the factors determining PD-L1 expression by both tumorinfiltrating lymphocytes and tumor cells in all breast cancer subtypes. It also suggests a potential role for immune checkpoint blockade therapy in primary invasive lobular carcinoma of the breast. Furthermore, our findings suggest that it may not be appropriate to use ER status, HER-2 status, or carcinoma grade to select patients for clinical trials evaluating immunotherapeutic strategies in patients with primary invasive lobular carcinoma. As invasive lobular breast carcinomas often respond less well to chemotherapy relative to ductal carcinomas, targeted immunotherapy may provide additional treatment options for a subset of patients with this histologic subtype of breast carcinoma.

\section{Acknowledgments}

This work was supported by funding from the Eggleston Award in Surgical Pathology (RAK), the Safeway-Avon Breast Center Pilot Project Award (ACM), and the Breast Cancer Research Foundation (BCRF; LAE).

\section{Disclosure/conflict of interest}

LAE receives research funding from Genentech, Roche, EMD Serono, Merck, Astra Zeneca, and Corvus. JMT receives research funding from BristolMyers Squibb, and is a consultant and member of the advisory board for Bristol-Meyers Squibb, Merck, and Astra Zeneca. The remaining authors declare no conflict of interest.

\section{References}

1 Mahmoud SMA, Lee AHS, Paish EC, et al. The prognostic significance of B lymphocytes in invasive carcinoma of the breast. Breast Cancer Res Treat 2012;132:545-553.

2 Denkert C, Loibl S, Noske A, et al. Tumor-associated lymphocytes as an independent predictor of response to neoadjuvant chemotherapy in breast cancer. J Clin Oncol 2010;28:105-113.

3 Bates GJ, Fox SB, Han C, et al. Quantification of regulatory $\mathrm{T}$ cells enables the identification of high-risk breast cancer patients and those at risk of late relapse. J Clin Oncol 2006;24:5373-5380.

4 Schalper KA, Velcheti V, Carvajal D, et al. In situ tumor PD-L1 mRNA expression is associated with increased TILs and better outcome in breast carcinomas. Clin Cancer Res 2014;20:2773-2782.

5 Loi S, Sirtaine N, Piette F, et al. Prognostic and predictive value of tumor-infiltrating lymphocytes in a phase III randomized adjuvant breast cancer trial in node-positive breast cancer comparing the addition of docetaxel to doxorubicin with doxorubicin-based chemotherapy: BIG 02-98. J Clin Oncol 2013;31:860-867.

6 Adams S, Gray RJ, Demaria S, et al. Prognostic value of tumor-infiltrating lymphocytes in triple-negative breast cancers from two phase III randomized adjuvant breast cancer trials: ECOG 2197 and ECOG 1199. J Clin Oncol 2014;32:2959-2966.

7 Loi S, Michiels S, Salgado R, et al. Tumor infiltrating lymphocytes are prognostic in triple negative breast cancer and predictive for trastuzumab benefit in early breast cancer: results from the FinHER trial. Ann Oncol 2014;25:1544-1550.

8 Kreike B, van Kouwenhove M, Horlings H, et al. Gene expression profiling and histopathological characterization of triple-negative/basal-like breast carcinomas. Breast Cancer Res 2007;9:R65.

9 Emens LA. Breast cancer immunobiology driving immunotherapy: vaccines and immune checkpoint 
blockade. Expert Rev Anticancer Ther 2012;12: 1597-1611.

10 Cimino-Mathews A, Foote JB, Emens LA. Immune targeting in breast cancer. Oncology (Williston Park) 2015;29:375-385.

11 Lipson EJ, Forde PM, Hammers HJ, et al. Antagonists of PD-1 and PD-L1 in cancer treatment. Semin Oncol 2015;42:587-600.

12 Cimino-Mathews A, Ye X, Meeker A, et al. Metastatic triple-negative breast cancers at first relapse have fewer tumor-infiltrating lymphocytes than their matched primary breast tumors: a pilot study. Hum Pathol 2013;44:2055-2063.

13 Dong H, Strome SE, Salomao DR, et al. Tumorassociated B7-H1 promotes T-cell apoptosis: a potential mechanism of immune evasion. Nat Med 2002;8: 793-800.

14 Mittendorf EA, Philips AV, Meric-Bernstam F, et al. PD-L1 expression in triple-negative breast cancer. Cancer Immunol Res 2014;2:361-370.

15 Gatalica Z, Snyder C, Maney T, et al. Programmed cell death 1 (PD-1) and its ligand (PD-L1) in common cancers and their correlation with molecular cancer type. Cancer Epidemiol Biomarkers Prev 2014;23:2965-2970.

16 Ghebeh H, Mohammed S, Al-Omair A, et al. The B7-H1 (PD-L1) T lymphocyte-inhibitory molecule is expressed in breast cancer patients with infiltrating ductal carcinoma: correlation with important high-risk prognostic factors. Neoplasia 2006;8:190-198.

17 Wimberly $\mathrm{H}$, Brown JR, Schalper $\mathrm{K}$, et al. PD-L1 expression correlates with tumor-infiltrating lymphocytes and response to neoadjuvant chemotherapy in breast cancer. Cancer Immunol Res 2015;3:326-332.

18 Cimino-Mathews A, Thompson E, Taube JM, et al. PD-L1 (B7-H1) expression and the immune tumor microenvironment in primary and metastatic breast carcinomas. Hum Pathol 2016;47:52-63.

19 Thompson E, Taube JM, Elwood H, et al. The immune microenvironment of breast ductal carcinoma in situ. Mod Pathol 2016;29:249-258.

20 Michaut M, Chin S-F, Majewski I, et al. Integration of genomic, transcriptomic and proteomic data identifies two biologically distinct subtypes of invasive lobular breast cancer. Sci Rep 2016;6:18517.

21 Tringler B, Zhuo S, Pilkington G, et al. B7-H4 is highly expressed in ductal and lobular breast cancer. Clin Cancer Res 2005;11:1842-1848.

22 Sabatier R, Finetti P, Mamessier E, et al. Prognostic and predictive value of PDL1 expression in breast cancer. Oncotarget 2015;6:5449-5464.

23 Liu C, Liu J, Wang J, et al. B7-H3 expression in ductal and lobular breast cancer and its association with IL-10. Mol Med Rep 2013;7:134-138.

24 Heaphy CM, Asch-Kendrick R, Argani P, et al. Telomere length alterations unique to invasive lobular carcinoma. Hum Pathol 2015;46:1197-1203.

25 Asch-Kendrick RJ, Samols MA, Lilo MT, et al. NKX3.1 is expressed in ER-positive and AR-positive primary breast carcinomas. J Clin Pathol 2014;67: 768-771.

26 Salgado R, Denkert C, Demaria S, et al. The evaluation of tumor-infiltrating lymphocytes (TILs) in breast cancer: recommendations by an International TILs Working Group 2014. Ann Oncol 2015;26:259-271.

27 Denkert C, Wienert S, Poterie A, et al. Standardized evaluation of tumor-infiltrating lymphocytes in breast cancer: results of the ring studies of the international immuno-oncology biomarker working group. Mod Pathol 2016;29:1155-1164.

28 Herbst RS, Soria J-C, Kowanetz M, et al. Predictive correlates of response to the anti-PD-L1 antibody MPDL3280A in cancer patients. Nature 2014;515: 563-567.

29 Ghebeh H, Barhoush E, Tulbah A, et al. FOXP3+ Tregs and $\mathrm{B} 7-\mathrm{H} 1+/ \mathrm{PD}-1+\mathrm{T}$ lymphocytes co-infiltrate the tumor tissues of high-risk breast cancer patients: Implication for immunotherapy. BMC Cancer 2008;8: 57.

30 Droeser R, Zlobec I, Kilic E, et al. Differential pattern and prognostic significance of CD4+, FOXP3+ and IL-17+ tumor infiltrating lymphocytes in ductal and lobular breast cancers. BMC Cancer 2012;12:134.

31 Loke P, Allison JP. PD-L1 and PD-L2 are differentially regulated by Th1 and Th2 cells. Proc Natl Acad Sci USA 2003;100:5336-5341.

32 Liu J, Hamrouni A, Wolowiec D, et al. Plasma cells from multiple myeloma patients express B7-H1 (PD-L1) and increase expression after stimulation with IFN\{gamma\} and TLR ligands via a MyD88-, TRAF6-, and MEK-dependent pathway. Blood 2007;110:296-304.

33 Kondo A, Yamashita T, Tamura H, et al. Interferongamma and tumor necrosis factor-alpha induce an immunoinhibitory molecule, B7-H1, via nuclear factor-kappaB activation in blasts in myelodysplastic syndromes. Blood 2010;116:1124-1131.

34 Qian Y, Deng J, Geng L, et al. TLR4 signaling induces B7-H1 expression through MAPK pathways in bladder cancer cells. Cancer Invest 2008;26:816-821.

35 Taube JM, Anders RA, Young GD, et al. Colocalization of inflammatory response with B7-h1 expression in human melanocytic lesions supports an adaptive resistance mechanism of immune escape. Sci Transl Med 2012;4:127ra37.

36 Lipson EJ, Vincent JG, Loyo M, et al. PD-L1 expression in the Merkel cell carcinoma microenvironment: association with inflammation, Merkel cell polyomavirus and overall survival. Cancer Immunol Res 2013;1: 54-63.

37 Velcheti V, Schalper KA, Carvajal DE, et al. Programmed death ligand-1 expression in non-small cell lung cancer. Lab Invest 2014;94:107-116.

38 Ritprajak P, Azuma M. Intrinsic and extrinsic control of expression of the immunoregulatory molecule PD-L1 in epithelial cells and squamous cell carcinoma. Oral Oncol 2015;51:221-228.

39 Parsa AT, Waldron JS, Panner A, et al. Loss of tumor suppressor PTEN function increases B7-H1 expression and immunoresistance in glioma. Nat Med 2007;13: 84-88.

40 Zhu J, Chen L, Zou L, et al. MiR-20b, -21, and -130b inhibit PTEN expression resulting in B7-H1 overexpression in advanced colorectal cancer. Hum Immunol 2014;75:348-353.

41 Green MR, Monti S, Rodig SJ, et al. Integrative analysis reveals selective 9p24.1 amplification, increased PD-1 ligand expression, and further induction via JAK2 in nodular sclerosing Hodgkin lymphoma and primary mediastinal large B-cell lymphoma. Blood 2010;116: 3268-3277.

42 Ansell SM, Lesokhin AM, Borrello I, et al. PD-1 blockade with nivolumab in relapsed or refractory Hodgkin's lymphoma. N Engl J Med 2015;372:311-319. 
43 Barrett MT, Anderson KS, Lenkiewicz E, et al. Genomic amplification of 9p24.1 targeting JAK2, PD-L1, and PD-L2 is enriched in high-risk triple negative breast cancer. Oncotarget 2015;6:26483-26493.

44 Marzec M, Zhang Q, Goradia A, et al. Oncogenic kinase NPM/ALK induces through STAT3 expression of immunosuppressive protein CD274 (PD-L1, B7-H1). Proc Natl Acad Sci USA 2008;105:20852-20827.

45 Yamamoto R, Nishikori M, Tashima M, et al. B7-H1 expression is regulated by MEK/ERK signaling pathway in anaplastic large cell lymphoma and Hodgkin lymphoma. Cancer Sci 2009;100:2093-2100.

46 Azuma K, Ota K, Kawahara A, et al. Association of PD-L1 overexpression with activating EGFR mutations in surgically resected nonsmall-cell lung cancer. Ann Oncol 2014;25:1935-1940.

47 Mani NL, Schalper KA, Hatzis C, et al. Quantitative assessment of the spatial heterogeneity of tumorinfiltrating lymphocytes in breast cancer. Breast Cancer Res 2016;18:78. 